\title{
FGD5-AS1 facilitates the osteogenic differentiation of human bone marrow- derived mesenchymal stem cells via targeting the miR-506-3p/BMP7 axis
}

Jun Li, Xingbiao Wu, Yaohua Shi and Hong Zhao*

\begin{abstract}
Background: Osteoporosis is a systemic disease characterized by impaired bone formation, increased bone resorption, and brittle bone fractures. The osteogenic differentiation of human bone marrow-derived mesenchymal stem cells (hBMSCs) is considered to be a vital process for bone formation. Numerous studies have reported that long non-coding RNAs (IncRNAs) are involved in the osteogenic differentiation of hBMSCs. The present study aimed to investigate the effect of FGD5 antisense RNA 1 (FGD5-AS1) on osteogenic differentiation.

Methods: RT-qPCR was performed to detect the expression of FGD5-AS1, miR-506-3p, and osteogenesis-related genes OCN, OPN, OSX, and RUNX2. Western blotting was carried out to detect the protein levels of osteogenesisrelated markers. In addition, the regulatory effect of FGD5-AS1 on osteogenic differentiation was detected through alkaline phosphatase (ALP) activity, Alizarin Red S (ARS) staining, and Cell Counting Kit-8 (CCK-8). Bioinformatics analysis and luciferase reporter assay were used to predict and validate the interaction between FGD5-AS1 and miR-506-3p as well as miR-506-3p and bone morphogenetic protein 7 (BMP7).

Results: The RT-qPCR analysis revealed that FGD5-AS1 was upregulated in hBMSCs following induction of osteogenic differentiation. In addition, FGD5-AS1 knockdown attenuated hBMSC viability and osteogenic differentiation. Bioinformatics analysis and luciferase reporter assays verified that FGD5-AS1 could directly interact with microRNA (miR)-506-3p. Furthermore, miR-506-3p could directly target the 3'-untranslated region (3'-UTR) of BMP7. Additionally, functional assays demonstrated that miR-506-3p silencing could restore the suppressive effect of FGD5-AS1 knockdown on osteogenic differentiation and viability of hBMSCs, and miR-506-3p could attenuate osteogenic differentiation via targeting BMP7.

Conclusions: Taken together, the results of the present study suggested that FGD5-AS1 could positively regulate the osteogenic differentiation of hBMSCs via targeting the miR-506-3p/BMP7 axis.
\end{abstract}

Keywords: FGD5-AS1, miR-506-3p, BMP7, Osteoporosis, BMSC

* Correspondence: hongzhao1982@163.com

Department of Spinal Surgery, Changzhou Hospital of Traditional Chinese

Medicine, No. 25 Heping North Road, Changzhou, Jiangsu 213000, P.R. China

(c) The Author(s). 2021 Open Access This article is licensed under a Creative Commons Attribution 4.0 International License, which permits use, sharing, adaptation, distribution and reproduction in any medium or format, as long as you give appropriate credit to the original author(s) and the source, provide a link to the Creative Commons licence, and indicate if changes were made. The images or other third party material in this article are included in the article's Creative Commons licence, unless indicated otherwise in a credit line to the material. If material is not included in the article's Creative Commons licence and your intended use is not permitted by statutory regulation or exceeds the permitted use, you will need to obtain permission directly from the copyright holder. To view a copy of this licence, visit http://creativecommons.org/licenses/by/4.0/. The Creative Commons Public Domain Dedication waiver (http://creativecommons.org/publicdomain/zero/1.0/) applies to the data made available in this article, unless otherwise stated in a credit line to the data. 


\section{Introduction}

Osteoporosis is a chronic inflammatory bone disease characterized by the destruction of the bone trabecular structure and reduced bone density [1]. Due to the aging population, the incidence of osteoporosis in the elderly population is increasing [2], posing a major socioeconomic burden. Previous studies have shown that osteoporosis is mainly caused by attenuated osteogenic differentiation of human bone marrow-derived mesenchymal stem cells (hBMSCs) [3]. Therefore, understanding the regulatory mechanism underlying the differentiation of hBMSCs is crucial for the development of novel treatment approaches to osteoporosis.

Long non-coding RNAs (lncRNAs) are non-coding RNAs, $>200$ nt in length [4]. Numerous studies have reported that lncRNAs serve pivotal roles in regulating various biological processes, such as stem cell selfrenewal and differentiation $[5,6]$. More specifically, several studies have reported that lncRNAs are involved in the pathogenesis of osteoporosis. For instance, lncRNA growth arrest-specific transcript 5 (GAS5) may improve osteoporosis through accelerating MSC osteogenic differentiation via the microRNA (miR)-498/runt-related transcription factor 2 (RUNX2) axis [7]. Additionally, lncRNA nuclear-enriched abundant transcript 1 (NEAT1) could facilitate hBMSC osteogenic differentiation via modulating the miR-29b-3p/bone morphogenetic protein 1 (BMP1) axis [8]. Another study showed that IncRNA MALAT1 could suppress the osteogenic differentiation of MSCs in osteoporotic rats via the MAPK signaling [9]. LncRNA FGD5 antisense RNA 1 (FGD5-AS1) has been reported to enhance osteosarcoma cell proliferation and migration [10]. Recently, Yang et al. demonstrated that FGD5-AS1 expression was downregulated in cartilage tissues of patients with osteoarthritis, and knockdown of FGD5-AS1 inhibited the viability of chondrocytes but promoted apoptosis in osteoarthritis [11]. However, the molecular mechanisms of FGD5-AS1 in hBMSC differentiation remain unclear.

miRNAs are a class of small non-coding RNAs, 20-25 nt in length [12]. They can inversely modulate the expression levels of target genes via binding to the 3 '-untranslated region $\left(3^{\prime}\right.$-UTR) of mRNA and further degrade target mRNAs or inhibit mRNA translation [13, 14]. Emerging evidence has suggested that miRNAs can modulate osteogenic differentiation and bone formation $[15,16]$. The suppressive effect of miR-506-3p has been verified in several types of cancer $[17,18]$. Moreover, miR-506-3p could inhibit osteosarcoma cell proliferation and metastasis by regulating RAB3D [19]. However, its function and regulatory mechanism in osteogenic differentiation have not been previously reported. BMP7, also known as osteogenic protein 1 (OP-1), is a member of the BMP family, which plays a crucial role in osteogenesis and chondrogenesis [20, 21]. A previous study revealed that miR-506-3p downregulated BMP7 to modulate osteoblast viability, differentiation, and migration [22]. However, the specific biological role of the miR-506-3p/BMP7 axis in osteoporosis remains unknown.

The present study aimed to investigate the effect of the FGD5-AS1/miR-506-3p/BMP7 axis on the osteogenic differentiation of BMSCs.

\section{Materials and methods \\ Clinical samples}

Bone tissues were collected from patients with osteoporosis ( $n=17$; female, 8 ; male, 9; age, $51-75$ years) and healthy controls $(n=17$; female, 8 ; male, 9; age, 50-74 years) at Changzhou Hospital of Traditional Chinese Medicine. The inclusion criteria for patients were as follows: (i) patients provided informed consent and (ii) patients were diagnosed with osteoporosis. Patients with complications due to other diseases, such as chronic inflammatory diseases (such as inflammatory bowel disease and rheumatoid arthritis), were excluded. The present study was approved by the Ethics Committee of Changzhou Hospital of Traditional Chinese Medicine (approval no. CTC20191103A07). Written informed consent was obtained from all patients prior to study enrollment.

\section{Cell culture}

hBMSCs were purchased from the American Type Culture Collection (Catalog number: PCS-500-012) and cultured in DMEM (HyClone; GE Healthcare Life Sciences) supplemented with 10\% FBS (Gibco; Thermo Fisher Scientific, Inc.), $100 \mathrm{mg} / \mathrm{l}$ streptomycin, and $100 \mathrm{U} / \mathrm{l}$ penicillin at $37{ }^{\circ} \mathrm{C}$ in a humidified $5 \% \mathrm{CO}_{2}$ atmosphere. To promote osteogenic differentiation, hBMSCs were maintained in osteogenic medium (OM) with dexamethasone $(100 \mathrm{nM})$ and $\beta$-glycerophosphate $(2 \mathrm{mM})$ for 14 days. The medium was replaced every 3 days.

\section{Cell transfection}

Short hairpin RNAs (shRNAs) targeting FGD5-AS1 (shFGD5-AS1) or BMP7 (shBMP7), scramble shRNAs (shNC), miR-506-3p mimic (5'-CAAAGU GCUUAC AGUGCAGGUAG-3'), mimics NC (5'-GUCCUGAG AAGGCUAGCA UAGAU-3'), miR-506-3p inhibitor (5'-CUACCUGCACUGUAAGCACUUUG-3'), and inhibitor NC (5'-CUAUGCUAGCCUUCUCAGGACUU$\left.3^{\prime}\right)$ were designed and synthesized by Shanghai GenePharma Co., Ltd., and were transfected into hBMSCs using Lipofectamine 3000 (Invitrogen; Thermo Fisher Scientific, Inc.). Following transfection for $48 \mathrm{~h}$, the cells were used for subsequent experiments. 


\section{RT-qPCR assay}

Total RNA was extracted from hBMSCs using TRIzol $^{\circ}$ Reagent (Invitrogen; Thermo Fisher Scientific, Inc.) and was reverse-transcribed into cDNA with the PrimeScript RT Reagent Kit (Takara Biotechnology Co., Ltd.). Subsequently, qPCR was carried out on the ABI 7500 Fast Real-Time PCR system (Applied Biosystems; Thermo Fisher Scientific, Inc.) using the SYBR Green Technology (Takara Biotechnology Co., Ltd.). GAPDH and U6 served as internal controls. The $2^{-\Delta \Delta \mathrm{Cq}}$ method [23] was used to quantify the relative gene expression levels. The primer sequences were listed as follows: FGD5-AS1, forward 5'-AGTTTCTCTCTAGATT-GCCTT-3' and reverse 5' -ATTGACATGTTAGTGCCCTT-3'; miR-5063p, forward 5'-ACACTCATAAGGCACCCTTC-3' and reverse 5'-TCTACTCAGAAGGGGAGTAC-3'; BMP7, forward 5'-GCAGCACAATTTG GGAA-3' and reverse 5'-ACAGGTGTTTCGAGAACTGGC-3'; GAPDH, forward 5'-AACGTGTCAGTGGTGGACCTG-3' and reverse 5'-AGTGGGTGTCGCTGTTGAAGT-3'; and U6, forward 5 '-CTCGCTTCGGCAGCACA-3' and reverse 5'-AACGCTTCACGAATTTGCGT-3'.

\section{Cell Counting Kit-8 (CCK-8) assay}

Cell viability was assessed using a CCK-8 assay (Dojindo Molecular Technologies, Inc.). Briefly, hBMSCs were seeded into a 96-well plate at a density of $5 \times 10^{3}$ cells/ well. Subsequently, $10 \mu \mathrm{l}$ of the CCK- 8 solution was added to each well, and cells were incubated at $37^{\circ} \mathrm{C}$ for $2 \mathrm{~h}$. The absorbance at $450 \mathrm{~nm}$ was used to evaluate the cell proliferation ability using a microplate reader (BioRad Laboratories, Inc.).

\section{Alkaline phosphatase (ALP) staining and activity assay}

ALP activity was assessed using an ALP activity kit (Beyotime) according to the manufacturer's protocol. The absorbance at a wavelength of $450 \mathrm{~nm}$ was measured using a microplate reader (Bio-Tek Instruments, Inc.). For ALP staining, the cells were washed three times with PBS and fixed with $4 \%$ paraformaldehyde for $30 \mathrm{~min}$ at room temperature. The ALP stain was performed with a BCIP/NBT ALP color development kit (Beyotime).

\section{Alizarin Red S (ARS) staining}

hBMSCs were fixed with $4 \%$ paraformaldehyde and stained with $2 \%$ ARS (Sigma-Aldrich) for $15 \mathrm{~min}$. Subsequently, cells were washed with PBS and observed under a light microscope (Leica).

\section{Western blotting}

Total proteins were extracted from hBMSCs using a RIPA lysis buffer (Sigma-Aldrich; Merck KGaA) containing protease inhibitors, and the protein concentration was measured by a Pierce BCA Protein Assay Kit (Thermo Fisher Scientific, Inc.). Subsequently, the total protein extracts were separated by $10 \%$ SDS-PAGE and transferred onto the PVDF membranes. Following blocking with $5 \%$ skimmed milk at $37{ }^{\circ} \mathrm{C}$ for $1 \mathrm{~h}$, the membranes were incubated with primary antibodies against OCN (1:1000; Abcam), OPN (1:1000; Abcam), OSX (1: 1000; Abcam), RUNX2 (1:1000; Abcam), and GAPDH (1:1000; Abcam) at $4{ }^{\circ} \mathrm{C}$ overnight. Then, the membranes were incubated with HRP-conjugated secondary antibodies for $2 \mathrm{~h}$. The bands were visualized using an ECL detection reagent (Invitrogen; Thermo Fisher Scientific, Inc.) and analyzed by the ImageJ software (National Institutes of Health).

\section{RNA immunoprecipitation (RIP) assay}

RIP assay was performed utilizing the EZ-Magna RIP kit (EMD Millipore). Briefly, hBMSCs were lysed in lysis buffer, and cell lysates were incubated with magnetic beads conjugated with anti-Ago 2 or anti-IgG antibodies overnight at $4{ }^{\circ} \mathrm{C}$. The samples were then treated with proteinase K. Following washing, the retrieved RNA was analyzed by RT-qPCR assay.

\section{Bioinformatics analysis and luciferase reporter assay}

StarBase v2.0 (http://starbase.sysu.edu.cn/) was used to predict the binding sites between miR-506-3p and FGD5-AS1 or BMP7. The 3'-UTR of FGD5-AS1-wildtype (WT), FGD5-AS1-mutant (MUT), BMP7-WT, and BMP7-MUT were sub-cloned into pmirGLO luciferase vectors (Promega Corporation). Subsequently, hBMSCs were co-transfected with the above plasmids and miR506-3p or NC mimics using Lipofectamine ${ }^{\text {tx }} 3000$ (Invitrogen; Thermo Fisher Scientific, Inc.). Following transfection for $48 \mathrm{~h}, \mathrm{hBMSCs}$ were harvested, and the luciferase activity was detected using a Dual-Luciferase Reporter Assay System (Promega Corporation). Firefly luciferase activity was normalized to Renilla (Promega Corporation) luciferase gene activity.

\section{Statistical analysis}

Data are expressed as the mean \pm SD. All statistical analyses were carried out using the SPSS 21.0 software (IBM Corp.). Statistical differences between the two groups were determined by Student's $t$-test, while those among multiple groups with one-way ANOVA were followed by Tukey's post hoc test. $P<0.05$ was considered to indicate a statistically significant difference.

\section{Results}

Levels of FGD5-AS1, miR-506-3p, and BMP7 after osteogenic differentiation of BMSCs

RT-qPCR assay results demonstrated that the FGD5AS1 expression levels were significantly higher in non- 

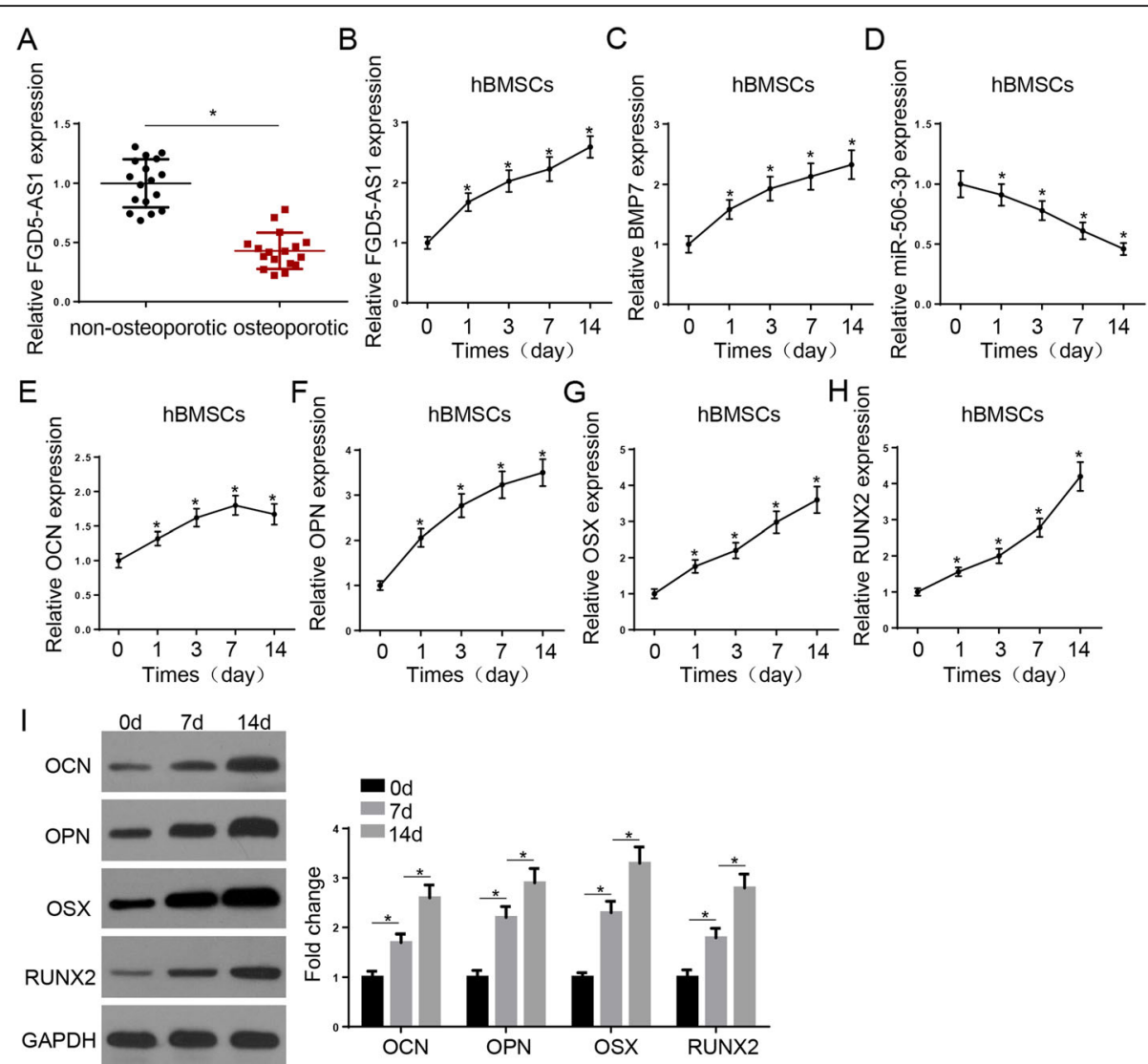

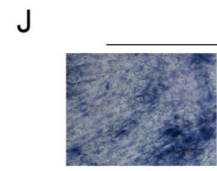

Od

$\mathrm{L}$

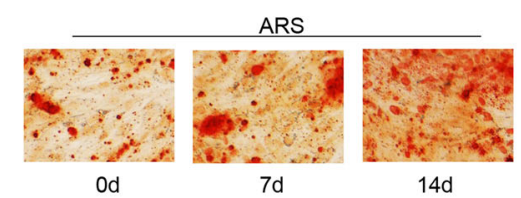

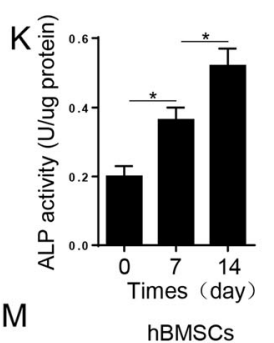

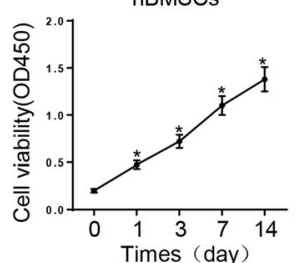

Fig. 1 Levels of FGD5-AS1, miR-506-3p, and BMP7 after osteogenic differentiation of BMSCs. A RT-qPCR was used to detect the expression of FGD5AS1 in bone tissues from patients with osteoporosis and healthy individuals. B-H RT-qPCR analysis was used to detect the expression of FGD5-AS1, miR-506-3p, BMP7, and osteogenesis-related genes (RUNX2, OCN, OSX, and OPN) in hBMSCs treated with OM for 14 days. I Western blotting assay showed the protein levels of osteogenesis-related genes in hBMSCs treated with osteogenic medium (OM) for 14 days. J, K ALP activity and ALP staining assays revealed the ALP activity of hBMSCs treated with OM for 14 days. $\mathbf{K}$ Alizarin Red staining showed the cell mineralization ability of hBMSCs treated with OM for 14 days. $\mathbf{M}$ CCK-8 assay showed the viability of hBMSCs during the process of osteogenic differentiation. ${ }^{*} P<0.05$

osteoporotic tissues compared with those in tissues from patients with osteoporosis (Fig. 1A). For the induction of osteogenic differentiation, hBMSCs were cultured in OM for 14 days. RT-qPCR indicated that FGD5-AS1 and BMP7 were upregulated from the first day of the induction of hBMSC osteogenic differentiation, whereas miR- 506-3p was downregulated after induction of osteogenic differentiation (Fig. 1B-D). Moreover, RT-qPCR analysis revealed that the expression of the osteogenesis-related genes OCN, OPN, OSX, and RUNX2 was notably upregulated (Fig. 1E-H). Consistently, western blot analysis showed that the protein levels of RUNX2, OCN, OSX, 
and OPN were increased following the culture of hBMSCs in OM (Fig. 1I). ALP staining, ALP activity, and ARS staining assays also showed that the osteogenic differentiation of hBMSCs in OM enhanced ALP staining, ALP activity, and mineralization ability of BMSCs (Fig. 1J-L). Furthermore, the CCK-8 assay demonstrated that hBMSC viability gradually increased during the osteogenic differentiation process (Fig. 1M). Collectively, these findings indicated that FGD5-AS1 could regulate the osteogenic differentiation of hBMSCs.

\section{FGD5-AS1 knockdown attenuates the osteogenic differentiation of hBMSCs}

To elucidate the effect of FGD5-AS1 on the osteogenic differentiation of hBMSCs, cells were transfected with shFGD5-AS1 to effectively silence the expression of FGD5-AS1 (Fig. 2A). In addition, the mRNA and protein expression levels of the osteogenic markers, OCN, OPN, OSX, and RUNX2, were reduced after FGD5-AS1 silencing (Fig. 2B, C). Furthermore, FGD5-AS1 depletion reduced the ALP activity and inhibited the mineralization ability in hBMSCs (Fig. 2D-F). Finally, the CCK-8 assay revealed that the hBMSC viability was attenuated by FGD5-AS1 silencing (Fig. 2G). The aforementioned data suggested that FGD5-AS1 knockdown could inhibit the osteogenic differentiation and viability of hBMSCs.

\section{miR-506-3p is directly targeted by FGD5-AS1}

Bioinformatics analysis, using the starBase website, predicted that miR-506-3p could be directly targeted by FGD5-AS1 (Fig. 3A). This finding was verified by a dualluciferase activity reporter assay, and the results demonstrated that miR-506-3p overexpression decreased the luciferase activity of FGD5-AS1-WT, while no changes were observed in cells transfected with FGD5-AS1-MUT (Fig. 3B). In addition, RT-qPCR analysis demonstrated that FGD5-AS1 knockdown increased the miR-506-3p expression (Fig. 3C). Finally, the expression levels of FGD5-AS1 were decreased following transfection of hBMSCs with miR-506-3p mimics (Fig. 3D). Taken together, these findings indicated that FGD5-AS1 could directly bind with miR-506-3p.

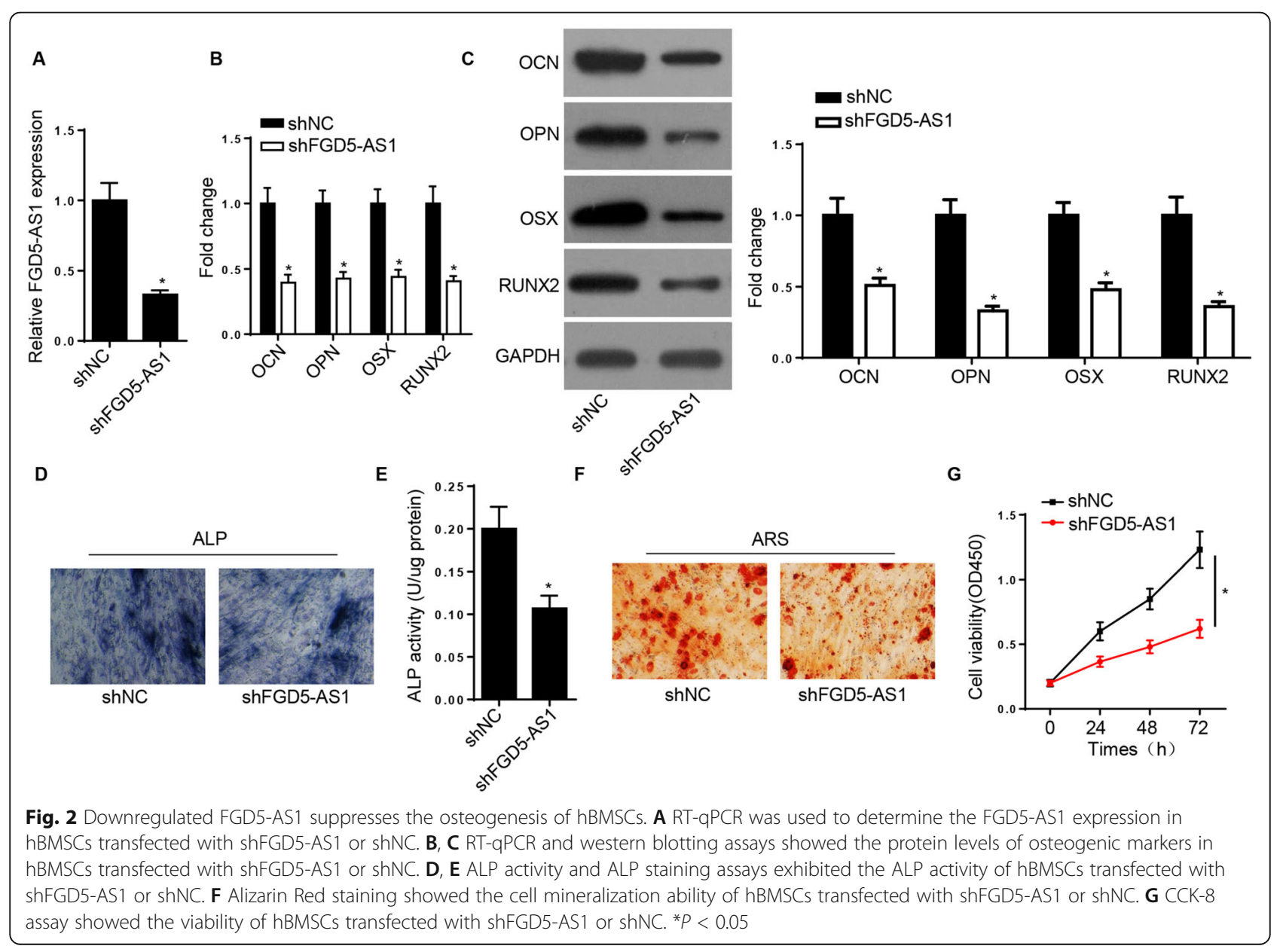




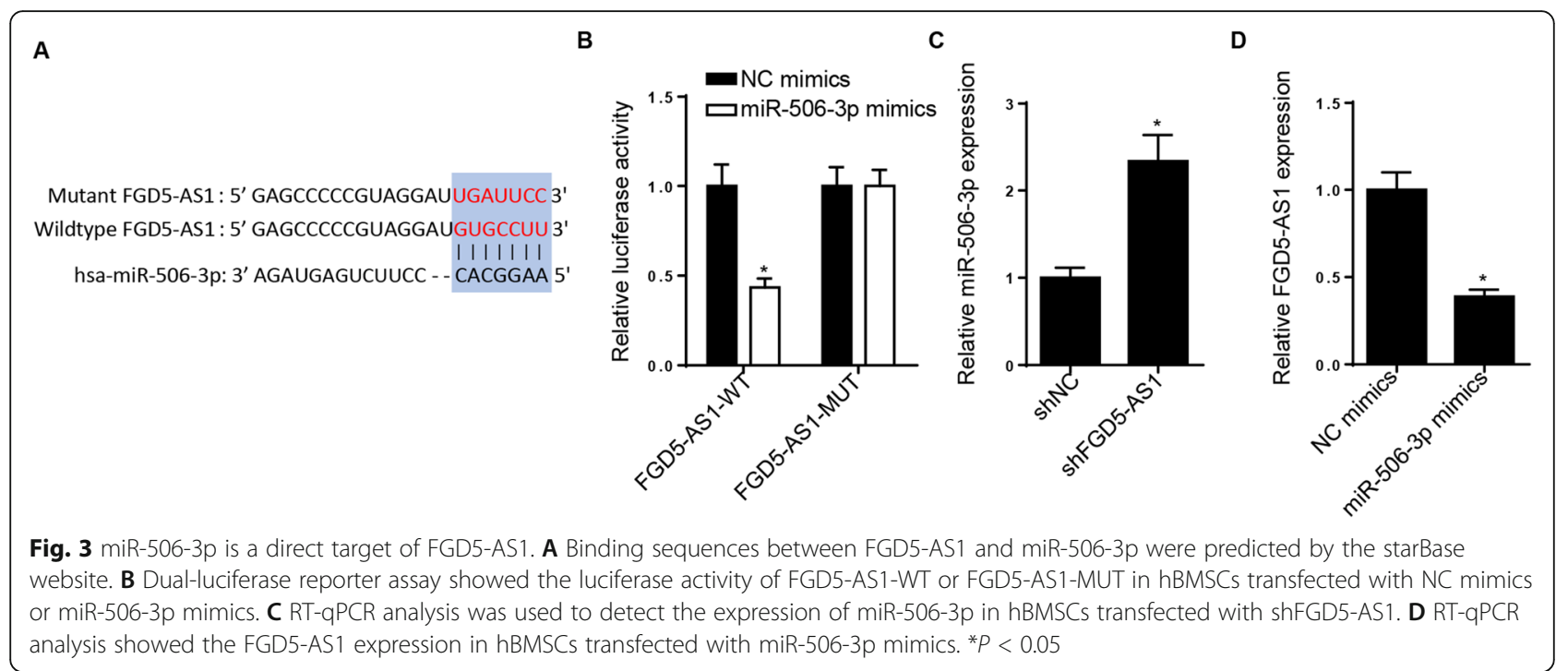

miR-506-3p silencing rescues the suppressive effect of FGD5-AS1 downregulation on the osteogenic differentiation of hBMSCs

Subsequently, we investigated whether FGD5-AS1 could regulate osteogenic differentiation via targeting miR506-3p. The transfection efficiency of the miR-506-3p inhibitor was confirmed by RT-qPCR (Fig. 4A). As shown in Fig. 4B, FGD5-AS1 knockdown reduced the expression of OCN, OPN, and RUNX2 in hBMSCs, while this effect was reversed after transfection of hBMSCs with miR-506-3p inhibitor. In addition, miR506-3p silencing rescued the reduced ALP activity and

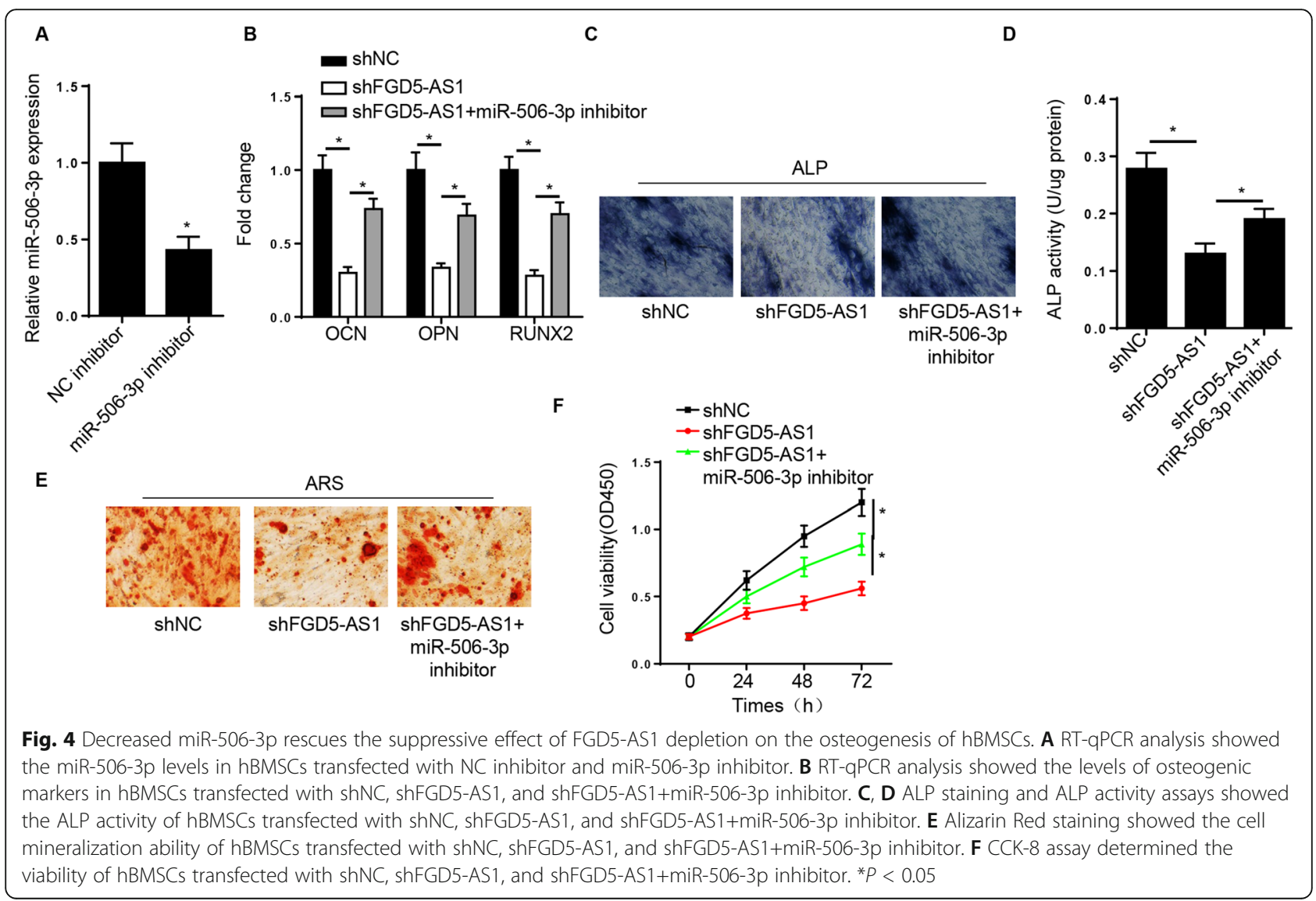


mineralized nodules mediated by FGD5-AS1 knockdown in hBMSCs (Fig. 4C-E). Additionally, the CCK-8 assay revealed that FGD5-AS1 knockdown attenuated the viability of hBMSCs, which was further promoted by miR506-3p downregulation (Fig. 4F). These data indicated that miR-506-3p silencing could rescue the suppressive effect of FGD5-AS1 downregulation on the osteogenic differentiation of hBMSCs.

FGD5-AS1 upregulates BMP7 expression via binding with miR-506-3p

The starBase website was utilized to predict the potential binding sites between miR-506-3p and BMP7. The putative binding sequences of miR-506-3p and BMP7 are shown in Fig. 5A. Subsequently, the luciferase reporter assay verified that miR-506-3p overexpression obviously decreased the luciferase activity of BMP7-WT. However, no significant changes were obtained in cells transfected with BMP7MUT (Fig. 5B). Additionally, RT-qPCR analysis showed that miR-506-3p mimics markedly downregulated BMP7 (Fig. 5C). Furthermore, RIP assays demonstrated that the expression levels of FGD5-AS1, miR-506-3p, and BMP7 were enriched in the anti-Ago2 group (Fig. 5D). Additionally, FGD5-AS1 depletion decreased the expression levels of BMP7 (Fig. 5E). Overall, these results indicated that FGD5-AS1 could modulate the BMP7 expression via sponging miR-506-3p.

\section{Silencing of BMP7 abrogates the promoting effect of the miR-506-3p inhibitor on the osteogenic differentiation of hBMSCs}

Subsequently, to investigate the role of BMP7 in promoting the FGD5-AS1-mediated osteogenic differentiation, shRNAs targeting BMP7 were synthesized to suppress the

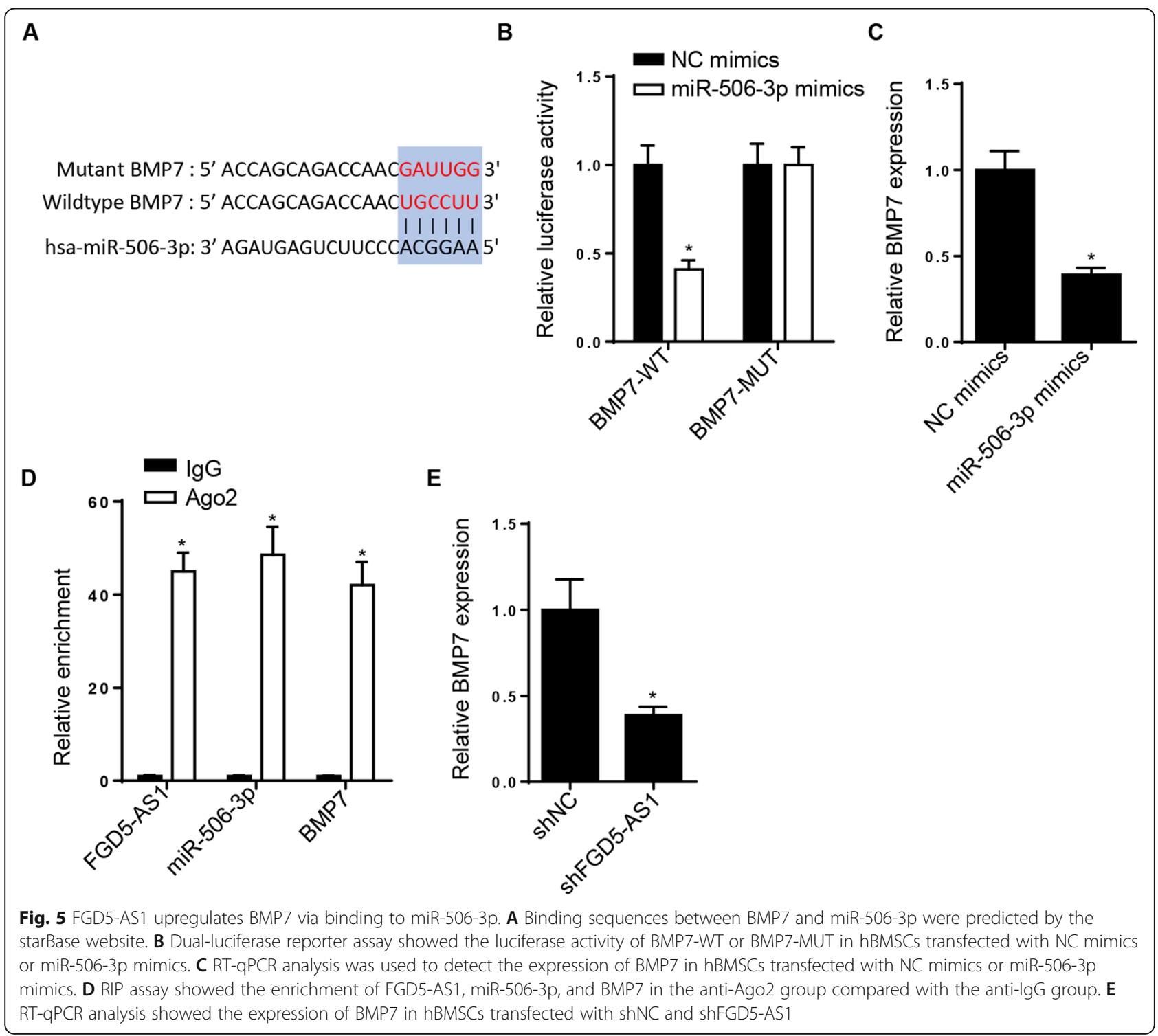


BMP7 expression (Fig. 6A). RT-qPCR analysis showed that miR-506-3p silencing increased the expression levels of OCN, OPN, and RUNX2 in hBMSCs, while BMP7 knockdown reversed this effect (Fig. 6B). In addition, BMP7 downregulation partially reversed the enhanced ALP activity, mineralized nodules, and cell viability caused by miR-506-3p inhibition (Fig. 6C-F). Overall, these results suggested that FGD5-AS1 could enhance osteogenic differentiation via regulating the miR-506-3p/BMP7 axis.

\section{Discussion}

Osteoporosis is an age-associated skeletal disease characterized by decreased bone mass and increased bone fragility and fracture risk [24]. An increasing number of individuals are currently suffering from osteoporosis [25]. Although progress has been made in the treatment of osteoporosis, the mechanisms involved in this disease remain unclear [26]. Previous studies have shown that hBMSCs have the potential of multidirectional differentiation and can be transformed into osteoblasts in response to appropriate stimuli [27]. Therefore, it is crucial to elucidate the regulatory mechanism underlying the osteogenic differentiation of hBMSCs.

Emerging evidence has suggested that lncRNAs serve vital roles in the progression of osteoporosis via regulating osteogenic differentiation. For example, a study demonstrated that IncRNA MSC-AS1 could inhibit the development of osteoporosis by promoting the osteogenic differentiation of BMSCs via regulating the miR-140-5p/ BMP2 axis [28]. HOTAIR was shown to suppress the osteogenic differentiation of BMSCs via modulating the Wnt/ $\beta$-catenin pathway [29]. Another study reported that lncRNA GAS5 accelerated osteogenic differentiation of BMSCs via targeting miR-135a-5p to regulate FOXO1 [30]. It has been reported that lncRNA FGD5 antisense RNA1 (FGD5-AS1) serves a crucial role in the progression of several types of malignant tumors [31, 32]. However, its effect on the osteogenic differentiation of hBMSCs remains unclear. Herein, the expression of FGD5-AS1 was upregulated during osteogenic differentiation. Furthermore, FGD5-AS1 silencing attenuated the osteogenic differentiation processes of hBMSCs.

Emerging evidence has suggested that lncRNAs act as sponges for miRNAs to modulate the expression of their target mRNAs [33]. Previous studies have indicated that miRNA may play a vital role in regulating the pathogenesis of various diseases [34-36]. Herein, we identified that miR-506-3p was a downstream target of FGD5-AS1. Moreover, miR-506-3p was upregulated by FGD5-AS1 depletion, while miR-506-3p overexpression reduced the expression of FGD5-AS1. Functional assays revealed that miR-506-3p downregulation reversed the suppressive effect of FGD5-AS1 silencing on ALP activity and viability of hBMSCs. Subsequently, BMP7 was identified as a direct target of miR-506-3p. Bone morphogenetic proteins (BMPs), as members of the transforming growth factor-

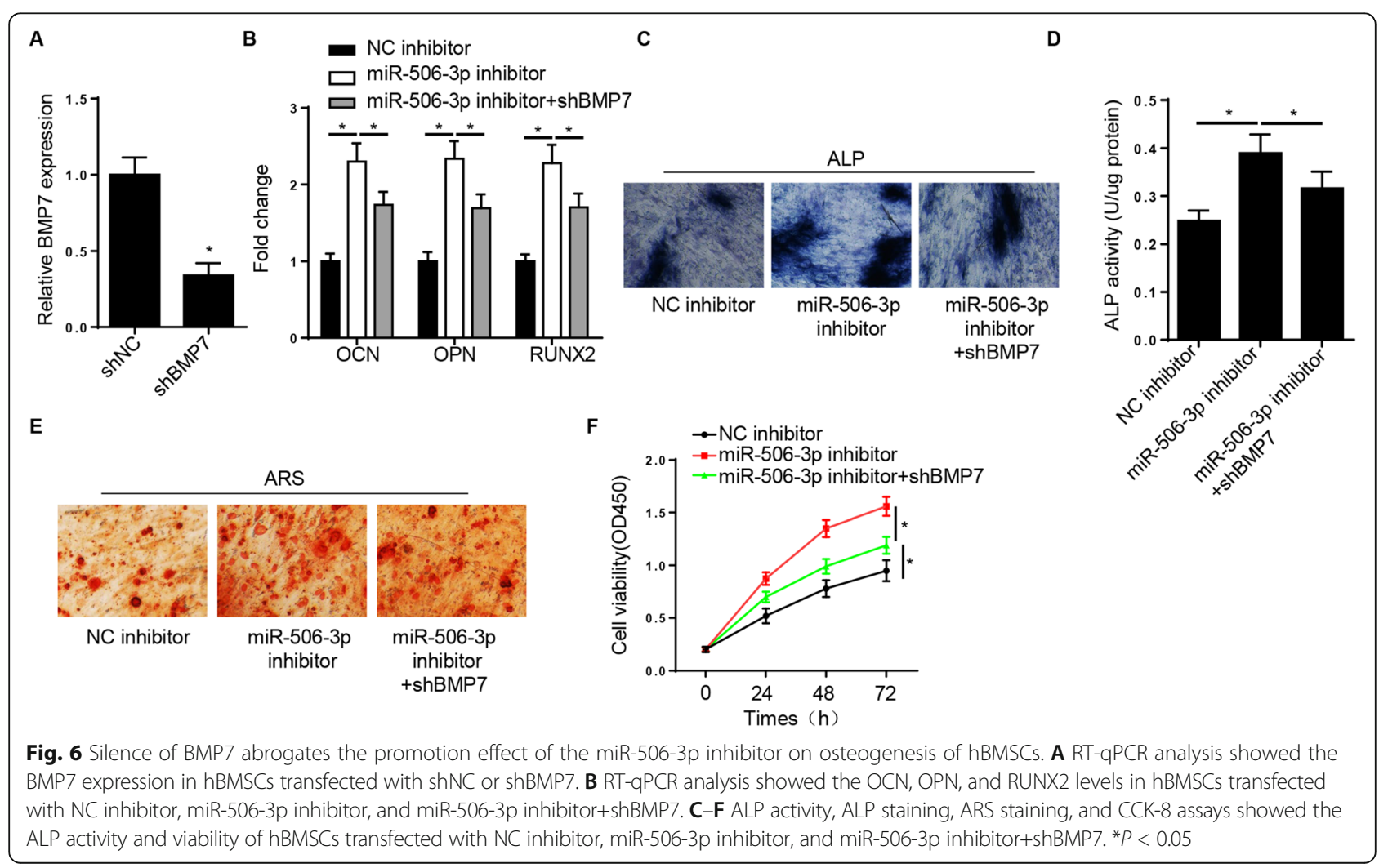


b (TGF-b) superfamily, play a vital role in inducing bone formation [37]. BMP-7 has been reported to stimulate a chondrogenic phenotype in adipose tissue-derived stem cells and enhance the chondrogenesis of synovial MSC when combined with TGF-b1 [38]. Moreover, Liu et al. reported that miR-542-3p modulated the osteogenic differentiation of vascular smooth muscle cells via targeting BMP7 [39]. Another study showed that BMP7 could promote osteogenic differentiation of human periosteal cells in vitro [40]. The results of the present study revealed that deletion of miR-506-3p increased ALP activity, mineralized nodules, and viability of hBMSCs, while BMP7 downregulation reversed these effects. The aforementioned findings suggested that FGD5-AS1 could promote the osteogenic differentiation of hBMSCs via the miR-506-3p/BMP7 axis.

\section{Conclusion}

To the best of our knowledge, the present study is the first to investigate the effect of FGD5-AS1 on the progression of osteogenic differentiation. The results demonstrated that FGD5-AS1 could promote the osteogenic differentiation of hBMSCs via regulating the miR-506$3 p / B M P 7$ axis, thus providing novel insights into the mechanisms underlying the osteogenic differentiation of hBMSCs. However, there are several factors affecting osteoporosis, while the present study mainly focused on the effects of osteogenic differentiation on osteoporosis. Therefore, further investigations are needed to uncover the possible involvement of other mechanisms in the regulation of osteoporosis.

\section{Abbreviations}

hBMSCs: Human bone marrow-derived mesenchymal stem cells; IncRNAs: Long non-coding RNAs; FGD5-AS1: FGD5 antisense RNA 1; ALP: Alkaline phosphatase; CCK-8: Cell Counting Kit-8; BMP7: Bone morphogenetic protein 7; 3'-UTR: 3'-Untranslated region; GAS5: Growth arrest-specific transcript 5; RUNX2: Runt-related transcription factor 2; BMP1: Bone morphogenetic protein 1; OP-1: Osteogenic protein 1; BMPs: Bone morphogenetic proteins; TGF-b: Transforming growth factor-b
\end{abstract}

\section{Acknowledgements}

Not applicable.

\section{Authors' contributions}

$J \mathrm{~L}$ and $\mathrm{XW}$ designed and carried out the study. YS and $\mathrm{HZ}$ participated in the experiments and statistical analysis. JL and $\mathrm{HZ}$ wrote the manuscript. $\mathrm{HZ}$ revised the manuscript. All authors read and approved the final manuscript.

\section{Funding}

Not applicable.

\section{Availability of data and materials}

The datasets used and/or analyzed during the current study are available from the corresponding author on reasonable request.

\section{Declarations}

Ethics approval and consent to participate

This study was approved by the Ethics Committee of the Changzhou Hospital of Traditional Chinese Medicine. All the patients signed a written informed consent.

\section{Consent for publication}

Not applicable.

\section{Competing interests}

The authors declare that they have no competing interests.

Received: 2 June 2021 Accepted: 25 August 2021

Published online: 12 November 2021

\section{References}

1. Qiao L, Liu D, Li CG, Wang YJ. MiR-203 is essential for the shift from osteogenic differentiation to adipogenic differentiation of mesenchymal stem cells in postmenopausal osteoporosis. Eur Rev Med Pharmacol Sci. 2018;22(18):5804-14. https://doi.org/10.26355/eurrev_201809_15906.

2. Chen IJ, Chiang CYF, Li YH, Chang CH, Hu CC, Chen DW, et al. Nationwide cohort study of hip fractures: time trends in the incidence rates and projections up to 2035. Osteoporos Int. 2015;26(2):681-8. https://doi.org/1 0.1007/s00198-014-2930-z.

3. Zhang Y, Liu Y, Wu M, Wang H, Wu L, Xu B, et al. MicroRNA-664a-5p promotes osteogenic differentiation of human bone marrow-derived mesenchymal stem cells by directly downregulating HMGA2. Biochem Biophys Res Commun. 2020;521(1):9-14. https://doi.org/10.1016/j.bbrc.2019. 09.122 .

4. Iyer MK, Niknafs YS, Malik R, Singhal U, Sahu A, Hosono Y, et al. The landscape of long noncoding RNAs in the human transcriptome. Nat Genet. 2015;47(3):199-208. https://doi.org/10.1038/ng.3192.

5. Chen X, Jiang C, Qin B, Liu G, Ji J, Sun X, et al. LncRNA ZNF503-AS1 promotes RPE differentiation by downregulating ZNF503 expression. Cell Death Dis. 2017:8(9):e3046. https://doi.org/10.1038/cddis.2017.382.

6. Zheng J, Yi D, Liu Y, Wang M, Zhu Y, Shi H. Long nonding RNA UCA1 regulates neural stem cell differentiation by controlling miR-1/Hes 1 expression. Am J Transl Res. 2017;9(8):3696-704.

7. Feng J, Wang JX, Li CH. LncRNA GAS5 overexpression alleviates the development of osteoporosis through promoting osteogenic differentiation of MSCs via targeting microRNA-498 to regulate RUNX2. Eur Rev Med Pharmacol Sci. 2019;23(18):7757-65. https://doi.org/10.26355/eurrev_201 909_18985

8. Zhang Y, Chen B, Li D, Zhou X, Chen Z. LncRNA NEAT1/miR-29b-3p/BMP1 axis promotes osteogenic differentiation in human bone marrow-derived mesenchymal stem cells. Pathol Res Pract. 2019;215(3):525-31. https://doi. org/10.1016/j.prp.2018.12.034.

9. Zheng $S$, Wang YB, Yang YL, Chen BP, Wang CX, Li RH, et al. LncRNA MALAT1 inhibits osteogenic differentiation of mesenchymal stem cells in osteoporosis rats through MAPK signaling pathway. Eur Rev Med Pharmacol Sci. 2019;23(11):4609-17. https://doi.org/10.26355/eurrev_201906_18038.

10. Li, C., et al., Long non-coding RNA FGD5-AS1 enhances osteosarcoma cell proliferation and migration by targeting miR-506-3p/RAB3D axis. 2021.

11. Yang Y, et al. FGD5-AS1 inhibits osteoarthritis development by modulating miR-302d-3p/TGFBR2 axis. Cartilage. 2021:19476035211003324.

12. Carthew RW, Sontheimer EJ. Origins and mechanisms of miRNAs and siRNAs. Cell. 2009;136(4):642-55. https://doi.org/10.1016/j.cell.2009.01.035.

13. Ambros V. The functions of animal microRNAs. Nature. 2004;431(7006):3505. https://doi.org/10.1038/nature02871.

14. Wahid F, Shehzad A, Khan T, Kim YY. MicroRNAs: synthesis, mechanism, function, and recent clinical trials. Biochim Biophys Acta. 2010;1803(11): 1231-43. https://doi.org/10.1016/j.bbamcr.2010.06.013.

15. Li H, Xie H, Liu W, Hu R, Huang B, Tan YF, et al. A novel microRNA targeting HDAC5 regulates osteoblast differentiation in mice and contributes to primary osteoporosis in humans. J Clin Invest. 2009;119(12):3666-77. https:// doi.org/10.1172/JCl39832.

16. Tamura M, et al. Canonical Wnt signaling activates miR-34 expression during osteoblastic differentiation. Mol Med Rep. 2013;8(6):1807-11. https://doi. org/10.3892/mmr.2013.1713. 
17. Liang TS, Zheng YJ, Wang J, Zhao JY, Yang DK, Liu ZS. MicroRNA-506 inhibits tumor growth and metastasis in nasopharyngeal carcinoma through the inactivation of the Wnt/beta-catenin signaling pathway by down-regulating LHX2. J Exp Clin Cancer Res. 2019;38(1):97. https://doi. org/10.1186/s13046-019-1023-4.

18. Li L, et al. Circ 100565 promotes proliferation, migration and invasion in non-small cell lung cancer through upregulating HMGA2 via sponging miR506-3p. Cancer Cell Int. 2020;20:160

19. Jiashi W, Chuang Q, Zhenjun Z, Guangbin W, Bin L, Ming H. MicroRNA-506$3 p$ inhibits osteosarcoma cell proliferation and metastasis by suppressing RAB3D expression. Aging (Albany NY). 2018;10(6):1294-305. https://doi.org/1 0.18632 /aging.101468.

20. Pizette S, Niswander L. BMPs are required at two steps of limb chondrogenesis: formation of prechondrogenic condensations and their differentiation into chondrocytes. Dev Biol. 2000;219(2):237-49. https://doi. org/10.1006/dbio.2000.9610.

21. Cheng $\mathrm{H}$, et al. Osteogenic activity of the fourteen types of human bone morphogenetic proteins (BMPs). J Bone Joint Surg Am. 2003;85(8):1544-52. https://doi.org/10.2106/00004623-200308000-00017.

22. Li X, Li K, Yu G, Yu C, Liu C. miR-342-5p inhibits expression of Bmp7 to regulate proliferation, differentiation and migration of osteoblasts. Mol Immunol. 2019;114:251-9. https://doi.org/10.1016/j.molimm.2019.07.027.

23. Livak KJ, Schmittgen TD. Analysis of relative gene expression data using real-time quantitative PCR and the $2^{-\Delta \Delta C T}$ method. Methods. 2001;25(4): 402-8. https://doi.org/10.1006/meth.2001.1262.

24. Kaperonis EA, Liapis CD, Kakisis JD, Dimitroulis D, Papavassiliou VG. Inflammation and atherosclerosis. Eur J Vasc Endovasc Surg. 2006;31(4):38693. https://doi.org/10.1016/j.ejvs.2005.11.001.

25. Wang O, Hu Y, Gong S, Xue Q, Deng Z, Wang L, et al. A survey of outcomes and management of patients post fragility fractures in China. Osteoporos Int. 2015;26(11):2631-40. https://doi.org/10.1007/s00198-015-3162-6.

26. Adler RA. Osteoporosis treatment: complexities and challenges. J Endocrinol Invest. 2016;39(7):719-20. https://doi.org/10.1007/s40618-016-0437-5.

27. Tuli R, Tuli S, Nandi S, Wang ML, Alexander PG, Haleem-Smith H, et al. Characterization of multipotential mesenchymal progenitor cells derived from human trabecular bone. Stem Cells. 2003;21(6):681-93. https://doi. org/10.1634/stemcells.21-6-681.

28. Zhang N, Hu X, He S, Ding W, Wang F, Zhao Y, et al. LncRNA MSC-AS promotes osteogenic differentiation and alleviates osteoporosis through sponging microRNA-140-5p to upregulate BMP2. Biochem Biophys Res Commun. 2019;519(4):790-6. https://doi.org/10.1016/j.bbrc.2019.09.058.

29. Shen JJ, Zhang CH, Chen ZW, Wang ZX, Yang DC, Zhang FL, et al. LncRNA HOTAIR inhibited osteogenic differentiation of BMSCs by regulating Wnt/ beta-catenin pathway. Eur Rev Med Pharmacol Sci. 2019;23(17):7232-46. https://doi.org/10.26355/eurrev_201909_18826.

30. Wang $X$, Zhao D, Zhu Y, Dong Y, Liu Y. Long non-coding RNA GAS5 promotes osteogenic differentiation of bone marrow mesenchymal stem cells by regulating the miR-135a-5p/FOXO1 pathway. Mol Cell Endocrinol. 2019;496:110534. https://doi.org/10.1016/j.mce.2019.110534.

31. Fan $Y$, et al. Long non-coding RNA FGD5-AS1 promotes non-small cell lung cancer cell proliferation through sponging hsa-miR-107 to up-regulate FGFR L1. Biosci Rep. 2020;40(1):BSR20193309.

32. Wu L, Zhu X, Song Z, Guo M, Liang J, Yan D. FGD5-AS1 facilitates glioblastoma progression by activation of Wnt/beta-catenin signaling via regulating miR-129-5p/HNRNPK axis. Life Sci. 2020;256:117998. https://doi. org/10.1016/j.lfs.2020.117998.

33. Zhu KP, Zhang CL, Ma XL, Hu JP, Cai T, Zhang L. Analyzing the interactions of mRNAs and ncRNAs to predict competing endogenous RNA networks in osteosarcoma chemo-resistance. Mol Ther. 2019;27(3):518-30. https://doi. org/10.1016/j.ymthe.2019.01.001.

34. Giordano L, Porta GD, Peretti GM, Maffulli N. Therapeutic potential of microRNA in tendon injuries. Br Med Bull. 2020;133(1):79-94. https://doi. org/10.1093/bmb/ldaa002.

35. Oliviero A, Della Porta G, Peretti GM, Maffulli N. MicroRNA in osteoarthritis: physiopathology, diagnosis and therapeutic challenge. Br Med Bull. 2019; 130(1):137-47. https://doi.org/10.1093/bmb/ldz015.

36. Gargano G, Oliviero A, Oliva F, Maffulli N. Small interfering RNAs in tendon homeostasis. Br Med Bull. 2021;138(1):58-67. https://doi.org/10.1093/bmb/ Idaa040.

37. Chimal-Monroy J, Rodriguez-Leon J, Montero JA, Gañan Y, Macias D, Merino $\mathrm{R}$, et al. Analysis of the molecular cascade responsible for mesodermal limb chondrogenesis: Sox genes and BMP signaling. Dev Biol. 2003;257(2):292301. https://doi.org/10.1016/S0012-1606(03)00066-6.

38. Miyamoto C, Matsumoto T, Sakimura K, Shindo H. Osteogenic protein-1 with transforming growth factor-beta1: potent inducer of chondrogenesis of synovial mesenchymal stem cells in vitro. J Orthop Sci. 2007;12(6):555-61. https://doi.org/10.1007/s00776-007-1176-4.

39. Liu H, Wang H, Yang S, Qian D. Downregulation of miR-542-3p promotes osteogenic transition of vascular smooth muscle cells in the aging rat by targeting BMP7. Hum Genomics. 2019;13(1):67. https://doi.org/10.1186/s4024 6-019-0245-Z.

40. Bei K, du Z, Xiong Y, Liao J, Su B, Wu L. BMP7 can promote osteogenic differentiation of human periosteal cells in vitro. Mol Biol Rep. 2012;39(9): 8845-51. https://doi.org/10.1007/s11033-012-1748-z.

\section{Publisher's Note}

Springer Nature remains neutral with regard to jurisdictional claims in published maps and institutional affiliations.
Ready to submit your research? Choose BMC and benefit from:

- fast, convenient online submission

- thorough peer review by experienced researchers in your field

- rapid publication on acceptance

- support for research data, including large and complex data types

- gold Open Access which fosters wider collaboration and increased citations

- maximum visibility for your research: over $100 \mathrm{M}$ website views per year

At BMC, research is always in progress.

Learn more biomedcentral.com/submissions 